\title{
RIE LAG IN HIGH ASPECT RATIO TRENCH ETCHING OF SILICON
}

\author{
Henri Jansen, Meint de Boer, Remco Wiegerink, Niels Tas, Edwin Smulders, Christina Neagu, and Miko Elwenspoek
}

MESA Research Institute, University of Twente, P.O. Box 217, 7500AE Enschede, The Netherlands

Phone: X-31-53-4892640; Secr.: X-31-53-4892751; Fax: X-31 53-4893343; E-mail: H.V.Jansen@eltn.utwente.nl

\begin{abstract}
While etching high aspect ratio trenches into silicon with reactive ion etching (RIE) using an $\mathrm{SF}_{6} / \mathrm{O}_{2}$ chemistry it is observed that the etch rate is depending on the mask opening. This effect is known as RIE lag and is caused by the depletion of etching ions and radicals or inhibiting neutrals during their trench passage. In order to decide which source is the main cause, we constructed special "horizontal trenches" where only radicals are controlling the etching. The experiment showed that radicals are not responsible for RIE lag. Inhibitor depletion will result in inverse RIE lag. This effect is not found during our experimentation which leaves us with ion depletion to explain RIE lag. Depletion of ions is caused by ions captured by the sidewalls due to the angular distribution of incoming ions into the trench opening and the deflection of ions in the trench due to electrostatic fields. The analysis given in this paper indicates that the influencing field causes ion deflection, ion depletion, and therefore RIE lag in micron-sized Si trenches for low-energetic ions. In all cases, thus independent of the feature size, the angular distribution of incoming ions is thought to have a major contribution to RIE lag at higher pressures. These phenomena will be treated theoretically and simulated using a program, written in $\mathrm{c}^{++}$under windows, in order to give a quantitative analysis of RIE lag.
\end{abstract}

\section{INTRODUCTION}

Etching high aspect ratio trenches (HART's) into silicon (Si) and other materials is becoming increasingly important for micro- and nano-engineering as in the case of e.g. combdriven structures, trench capacitors, and trench isolation for vertical transistors [1-3]. The aspect ratio (AR) is defined as the depth of the trench divided by its width. Currently, the most important technique to etch HART's is dry reactive ion etching (RIE). While etching HART's with RIE it is observed that the etch rate is depending on time and the mask opening. In general, smaller trench openings are etched slower than those which are wider (fig.la). This effect is known as RIE lag and seems to depend on the AR of the trench (i.e. AR scaling) rather than on the depth or width of the trench (i.e. feature size scaling). RIE lag is not only found in the conventional plasma reactors; dedicated machines equipped with high density sources and cryogenic cooling are facing identical problems. Moreover, the chemistry used to etch the sample and even the sample material is relatively unimportant with respect to RIE lag [4]. When the effect which is causing RIE lag is known, it is possible to minimise the decrease in etch rate with increasing $A R$ or decreasing feature size.

\section{PROBLEM DESCRIPTION}

RIE lag is clearly visible in the cross section of the grating shown in fig.1a. However, the RIE lag is nearly observed at the edge of the grating of fig. $1 \mathrm{~b}$. It is observed in fig. $1 \mathrm{~b}$, when we look into the grating, that the RIE lag is increasing fast. So, RIE lag is not only formed top-downwards the grating but also from sideways the grating.

There are three sources which may cause RIE lag; the flux of the etching ions and radicals and of the inhibiting neutrals which forms a passivating layer. When the flux of one of these streams of particles changes during its passage trough the trench RIE lag will be the result. It is difficult to decide which mechanism is responsible for the RIE lag observed in fig.1. Radical as well as ion depletion are candidates.

In the previous paper, the RIE lag was analysed qualitatively [5]. In this paper the RIE lag will be analysed quantitatively. First, a short overview of experiments carried out by other researchers will be given. Then, the inhibitor and radical depletion will be estimated as well as the apparently more pronounced ion depletion. Finally, the experiments techniques to find the mechanism(s) causing the lag effect will be treated.

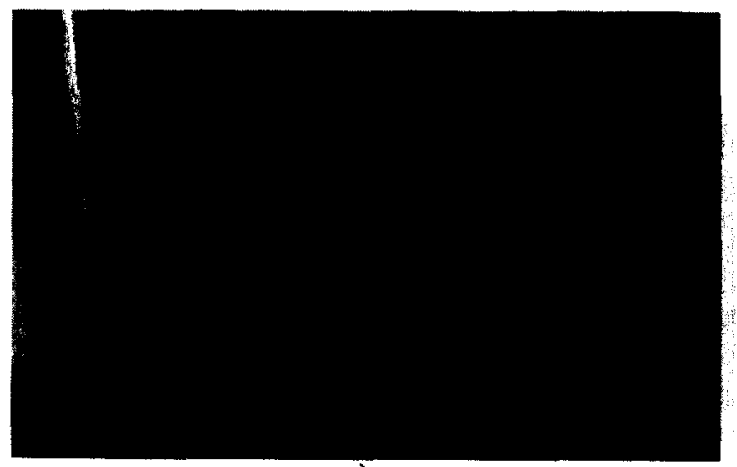

a)

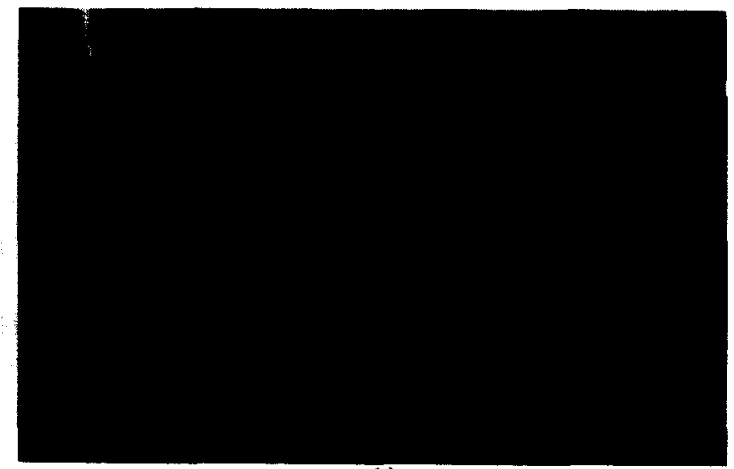

b)

Figure 1: RIE lag in the ICP-RIE. a) RIE-lag midways a grating pattern. b) RIE-lag free at the grating edge. 


\section{ION BEAM ASSISTED RADICAL ETCHING}

Reactive ion etching (RIE) or better: ion beam assisted radical etching (IBARE), enables the achievement of profile control due to the synergetic combination of physical sputtering with chemical activity of reactive species with high etch rate and high selectivity. Ion-enhanced etching can be divided into two main groups; ion-induced RIE (reaction-controlled etching) and ion-inhibitor RIE (desorption-controlled etching).

Ion-induced RIE is used when the substrate is not etched spontaneously, such as in the $\mathrm{Cl}_{2} / \mathrm{Si}$ or $\mathrm{O}_{2}$ /polymer system at room temperature. In ion-induced RIE a directional flux of ions coming from the plasma boundary region assists a random stream of radicals. In such processes, the yield per ion and the reaction probability of radicals depend on both the radical- and ion flux. This synergetic mechanism is expressed with a model proposed by Mayer and Barker based on Langmuir adsorption kinetics [6]. They assume that the etch rate $R$ is proportional to the surface coverage $\chi_{R}$ of the radicals times the ion energy $E_{1}$ and the ion flux $\phi_{1}$. The etch rate is also proportional to the number of bare sites $\left(1-\chi_{R}\right)$ on the surface times the radical flux $\phi_{R}$. Extracting $\chi_{R}$ from both equations results in an expression for the etch rate $R$ as a function of the ion energy-flux and radical sticking-flux

$$
\frac{1}{R}=\frac{1}{k_{R} \phi_{R} S_{R}}+\frac{1}{k_{I} \phi_{I} E_{I}}
$$

where $k_{1}$ is the volume removed per unit ion bombardment energy, $k_{R}$ is the volume removed per radical, and $S_{R}$ is the sticking probability of the radical on a bare surface. Note that the ion-radical synergism is clearly evident in this expression: In both cases when the ion or radical flux is small, the etch rate becomes vanishingly small.

In ion-inhibitor RIE the substrate is etched spontaneously, such as in the $\mathrm{SF}_{6} / \mathrm{Si}$ system. To achieve directionality, an inhibiting layer is needed, such as in the $\mathrm{SF}_{6} / \mathrm{O}_{2} / \mathrm{Si}$ system. Sidewalls of trenches are not exposed to ion bombardment and will be covered by this layer. However, the bottom of the trench is exposed to ion bombardment thus free from this deposit and etching can proceed. Therefore, the model of Mayer and Barker has to be modified to incorporate the inhibitor flux.

To find the etch rate as a function of the ion-, radical-, and inhibitor flux we start with the calculation of the surface coverage of the inhibitor $\chi_{\mathrm{P}}$ ( $\mathrm{p}=$ passivator). Per unit of time the number of inhibiting particles arriving from the gas phase and subsequently adsorbed should correspond to the desorption of the inhibitors which is proportional to the ion flux:

$$
\mathrm{k}_{\mathrm{I}} \phi_{\mathrm{I}} \mathrm{E}_{\mathrm{I}} * \chi_{\mathrm{P}}=\phi_{\mathrm{P}} \mathrm{S}_{\mathrm{P}} *\left(1-\chi_{\mathrm{P}}-\chi_{\mathrm{R}}\right)
$$

where $\phi_{\mathrm{P}}\left[\mathrm{mol} / \mathrm{m}^{2} \mathrm{~s}\right]$ the flux of inhibitors in the gas phase, $S_{\mathrm{p}}$ the probability of sticking of the inhibitor to the wafer surface, and $\left(1-\chi_{P}-\chi_{R}\right)$ the number of bare sites on the surface.

Per unit of time the number of radicals arriving from the gas phase at bare sides and sticking should match those which react with the surface giving rise to the etch rate:

$$
\mathrm{R}=\mathrm{R}_{\mathrm{S}} * \chi_{\mathrm{R}}=\mathrm{k}_{\mathrm{R}} \phi_{\mathrm{R}} \mathrm{S}_{\mathrm{R}} *\left(1-\chi_{\mathrm{P}}-\chi_{\mathrm{R}}\right)
$$

where $R_{S}$ is related to the surface reaction probability. The etch rate $R=R_{S}$ when the supply of radicals from the plasma phase is high enough to sustain a surface coverage of one.

Finally, eq. 2 and 3 are put into one equation resulting in

$$
\frac{1}{R}=\frac{1}{k_{R} \phi_{R} S_{R}}+\frac{1}{k_{I} \phi_{I} E_{I}} * \frac{\phi_{P} S_{P}}{k_{R} \phi_{R} S_{R}}+\frac{1}{R_{S}}
$$

The ion-inhibitor mechanism can be found from this expression by taking the following situations:

(1) When there is no flux of inhibitors $\left(\phi_{\mathrm{P}}=0\right)$ the etch rate becomes $1 / R=1 / k_{R} \phi_{R} S_{R}+1 / R_{S}$ which is indeed not depending on the ion flux. For a low radical flux $\left(\phi_{R} \Rightarrow 0\right)$, the etch rate approximates $R=k_{R} \phi_{R} S_{R}$ i.e. it is directly related to the radical flux (i.e. diffusion-controlled). At a high radical flux the etch rate will be $R=R_{S}$ i.e. it is depending only on the surface reaction mechanism and the supply of adsorbing radicals is sufficient (i.e. reaction-controlled).

(2) When the ion flux is vanishingly small $\left(\phi_{1} \Rightarrow 0\right)$ the etch rate is $R=k_{1} \phi_{1} E_{1}{ }^{*}\left(k_{R} \phi_{R} S_{R} / \phi_{P} S_{P}\right)$. So, the etch rate decreases when the flux of inhibitors increases with respect to the flux of radicals.

\section{LITERATURE}

The existence of RIE lag is found to be a notorious problem for micromechanics as well as for microelectronics [7]. In this chapter a comprehensive overview of recent literature on RIE lag will be given. In the excellent review from Gottscho eight mechanisms are considered to explain RIE lag: The first four are responsible for ion depletion and the last four for radical or inhibitor depletion [4]

(1) Differential charging of insulators,

(2) field curvature near conductors,

(3) image force deflection,

(4) ion shadowing with the ion angular distribution (IAD),

(5) radical/inhibitor shadowing,

(6) molecular flow,

(7) bulk diffusion, and

(8) surface diffusion.

Gottscho argues: "Three mechanisms, surface/bulk diffusion and field curvature, predict that for features with a given aspect ratio, RIE lag will increase with absolute feature size. This is not consistent with the observed scaling [9]........ In addition, bulk diffusion is not relevant for vacuum processing of micron-sized features because feature dimensions are much less than the gas-phase mean-free path. The image force mechanism predicts that RIE lag will increase as feature size decreases...... Nevertheless, it is expected to contribute to RIE lag in high aspect ratio, subquarter-micron trenches [8]. The relative importance of the remaining four mechanisms, molecular flow, ion/neutral shadowing, and differential insulator charging, consistent with aspect ratio scaling can be inferred by comparing observed trends with pressure, voltage, and ion flux to the scaling predictions for each mechanism."

In this paper we will re-examine these eight mechanisms and add some new. To two mechanisms, the image force and ion shadowing in corporation with the ion angular distribution function, extra attention have been paid. Since, these two mechanisms are believed to remain the most important mechanisms which cause RIE lag into $\mathrm{Si}$ using $\mathrm{SF}_{6} / \mathrm{O}_{2} \mathrm{RIE}$. 


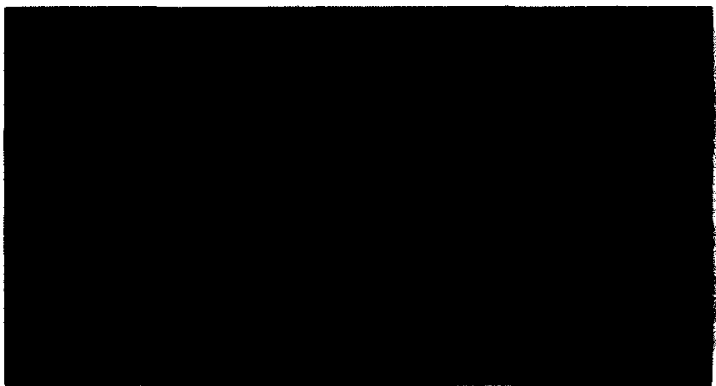

(a)

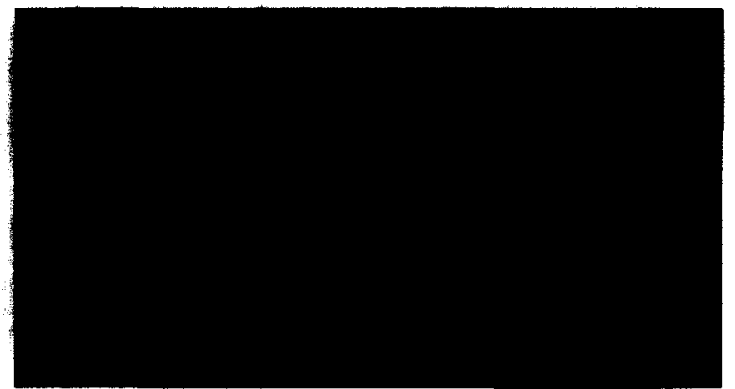

(b)

Figure 2: Horizontal trenches (i.e. sacrificial layer etching) showing that radical depletion is negligible. The AR 20/0.1 $=200$.

\section{EXPERIMENTAL}

In order to study RIE lag, two different RIE reactors were used, both using an $\mathrm{SF}_{6} / \mathrm{O}_{2}$ chemistry to etch $\mathrm{Si}$ samples. The first is a conventional RIE of the STS company used for the high pressure experiments ( $>50 \mathrm{~m}$ Torr). For the low pressure experiments a dedicated high density RIE is used build by the Plasma Technology company.

In eq-4 we arrived at the synergetic etch mechanism of radicals, ions, and inhibitors in ion-inhibitor RIE. From this equation we see that the depletion of inhibitors will result in a higher etch rate. So, in small trenches where the supply of inhibitors is restricted due to inhibitor shadowing, or other effects, inverse RIE lag should be the result. Because we never observed inverse RIE lag in our experiments, inhibitor depletion is excluded to be responsible for the lag effect during the RIE of silicon in an $\mathrm{SF}_{6} / \mathrm{O}_{2}$ chemistry. This might be expected beforehand because the same mechanisms which transport inhibitors in a trench, control the stream of radicals. So, the depletion of inhibitors could be counterbalanced by the depletion of radicals. Nevertheless, many mechanisms cause RIE lag and the "horizontal trench" experiment should distinguish between ion and radical depletion. After that, experimental data will be compared with the image force theory and the angular distribution simulation.

\subsection{Ion depletion versus radical depletion}

Gottscho used the kinetic model for ion-induced RIE, eq. 1, as a basis to describe the dependence of etching rates on aspect ratio [4]. However, the equations governing the ion-inhibitor processes are completely different (Eq.4). We assume that during the anisotropic etching of $\mathrm{Si}$ in an $\mathrm{SF}_{6} / \mathrm{O}_{2}$ chemistry the supply of radicals is always sufficient. So, the ions are controlling the etch process completely. The argument for this assumption is described next.

In order to decide which mechanism is the main cause for RIE lag, radical or ion depletion, we constructed special "horizontal trenches". Wafers were prepared with a thin layer of $100,300,400,1000$, and $2700[\mathrm{~nm}]$ of poly-Si respectively, sandwiched between two non-etching oxide layers. Before depositing the top oxide layer, the poly-Si was etched into a grating pattern with trench width of $2,4,6,8$, 10 , and $1000 \mu \mathrm{m}$ respectively. In this way, an array of extremely small prismatic tubes were fabricated. After breaking the sample the poly-Si was etched in a high pressure (200mTorr) $\mathrm{SF}_{6}$ plasma in the PE mode (Fig.2a). Under these circumstances ions and inhibitors are not important and only radicals are controlling the etching. We found the etch rate to be constant in time after $5,10,15$, and $20 \mu \mathrm{m}$ of etching for all the tubes. For example, the smallest tube $(100 \mathrm{~nm} * 2 \mu \mathrm{m})$ was etched at the same rate of ca. $1 \mu \mathrm{m} / \mathrm{min}$ as the biggest tube $\left(2700 \mathrm{~nm}^{*} 1000 \mu \mathrm{m}\right)$ up to aspect ratios over 100 . For example, fig. $2 \mathrm{~b}$ shows that radicals were able to underetch the $100 \mathrm{~nm} *$ $1000 \mu \mathrm{m}$ poly-Si layer $20[\mu \mathrm{m}]$ (i.e. the AR 20/0.1=200).

So, we conclude that the contribution of radical depletion on the RIE lag effect is small in the $\mathrm{SF}_{6} / \mathrm{O}_{2} / \mathrm{Si}$ system.

\subsection{Ion energy and angular distribution}

Now the radical depletion has been excluded, the depletion of ions will be considered. First, it can be estimated that the interactions between ions travelling in the trench at the same time is negligible and differential charging effects can be skipped because $\mathrm{Si}$ is a relatively good conductor [14]. The thickness of the dark space region $(\sim 1-10 \mathrm{~mm})$ is much bigger than the micron-sized structures and therefore the field distortion is also excluded. So, there are only two candidates left to explain the lag effect in our case: the image force mechanism and ion angular distribution in corporation with the ion shadowing effect. We will start with a simulation of the ion depletion due to the etching or capturing of ions at the trench sidewalls caused by the IAD. After that, the analytical results are given for a simple approximated IAD.

Finite element analysis: For our experiment and simulation we used the data given by Liu [12]. Liu measured the angular as well as the energy distribution of argon ions as a function of the pressure by a small orifice in the cathode of an RIE apparatus. Trenches were etched at the specific pressure and the corresponding data on the distribution functions was used in a simulation [14]. The data we used as input parameters in the simulator was taken from the pressure dependent IAD argon RIE sampling experiment (fig.9 from ref.12) where Liu measured the fraction of ions as a function of the incident angle. Of course, we have to multiply the flux distribution at these angles with the average energy to find the total energy-flux to the substrate as a function of the angle. We use fig. 14 [12] and we find the average ion energy to decrease with increasing incident angle. From these figures we extracted the data as found in the caption of fig. 3 .

The result of our simulation is shown in fig. 3 and it indicates that bottling is a typical result of the angular distribution of ions and predicts the lag effect. So, our results are in agreement with Jurgensen and his experiments [11]. Moreover, this time we used the measured distribution functions from literature to simulate the profile development of the trench which largely enhances the model's validity. 

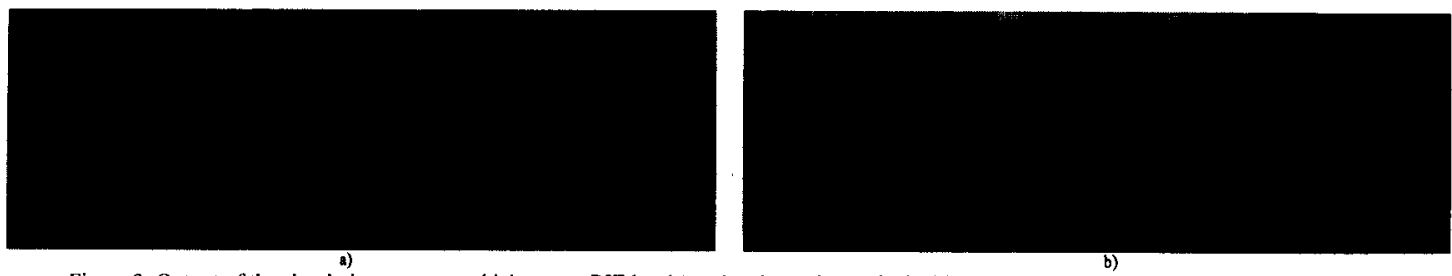

Figure 3: Output of the simulation program which causes RIE lag (a) and real trenches etched with the help of a conventional-RIE at $50 \mathrm{mT}$ Torr (b). The relative ion energy-flux coming in at a certain angle was settled at: $\{$ ion energy-flux/incident angle $\}=$

$\left\{13 / 0^{\circ}, 13 / 1^{\circ}, 13 / 2^{\circ}, 12 / 3^{\circ}, 12 / 4^{\circ}, 11 / 5^{\circ}, 9 / 6^{\circ}, 8 / 7^{\circ}, 6 / 8^{\circ}, 5 / 9^{\circ}, 5 / 10^{\circ}, 4 / 11^{\circ}, 4 / 12^{\circ}, 4 / 13^{\circ}, 3 / 14^{\circ}, 3 / 15^{\circ}, 3 / 16^{\circ}, 3 / 17^{\circ}, 2 / 18^{\circ}, 2 / 19^{\circ}\right\}$.

Theoretical analysis: Now, after the simulation result have been shown with the help of a finite element program, it is useful to give a theoretical analysis for a simple triangleshaped IAD and trench geometry [14]. For all trenches, the etch rate is almost the same until the shadowed incident angle equals the full-width of half-maximum (FWHM) of the IAD. This critical angle $\theta_{c}$ is directly related to the critical aspect ratio $A R_{c}$ of the circular hole as:

$$
1 / \mathrm{AR}_{\mathrm{c}}=2 \tan \left(\theta_{\mathrm{c}}\right) \sim 2 \tan (\mathrm{FWHM})
$$

Generally, for etching at low pressures the FWHM is fairly small $(<0.1 \mathrm{rad})$ and in such cases we may use $\tan (\mathrm{FWHM})$ FWHM:

$$
\mathrm{AR}_{\mathrm{c}} \sim 1 / 2 \mathrm{FWHM}
$$

After reaching $\mathrm{AR}_{\mathrm{c}}$ the etch rate will decrease due to ion shadowing. If we assume the IAD to decrease linearly per cone shell within two times its FWHM, then the etch rate will decrease inversely proportional with the AR. So, finally we are able to write down the relative etch rate of a hole as a function of the AR and the FWHM of the IAED, that is:

$$
\operatorname{RelR}=\left\{\begin{array}{ccc}
1 & \text { for } & \mathrm{AR}<\mathrm{AR}_{\mathrm{c}} \\
\mathrm{AR}_{\mathrm{c}} / \mathrm{AR} & \text { for } & \mathrm{AR}>\mathrm{AR}_{\mathrm{c}}
\end{array}\right.
$$

So, for small holes the lag effect is already pronounced because at the mid of its bottom it is depleted from ions due to ion shadowing. For the bigger trenches, the etch rate in the midpoint is still at its maximum because the ions from the whole IAD may arrive. Nevertheless, close to the sidewall the etch rate is smaller because of this shadowing. For holes etched with a triangle-like IAED and a FWHM of $5^{\circ}$, RIE lag will show up when the aspect ratio exceeds $A R=1 / 2 \tan ^{-1}\left(5^{\circ}\right)$ $=5.7$. For a hole of $3 \mu \mathrm{m}$ this means a depth of $17 \mu \mathrm{m}$ and for the $7 \mu \mathrm{m}$ opening the lag will start after a depth of $40 \mu \mathrm{m}$ has been reached. With the help of eq. 7 and $\delta h / \delta t=R_{\max }{ }^{*}$ RelR it is possible to find the etch-depth $h$ as a function of time:

$$
h=\left\{\begin{array}{cl}
R_{\max } * t & \text { for } t<t_{c} \\
R_{\max } * t_{c} \sqrt{\left(2 t / t_{c}-1\right)} & \text { for } t>t_{c}
\end{array}\right.
$$

where $t_{c}=h_{c} / R_{\max }$ and $h_{c}=w^{*} A R_{c}$. So, the etch rate ratio between two holes with width $w_{1}$ and $w_{2}$ is equal to: $h_{1} / h_{2}=$ $\left(t_{c 1} / t_{c 2}\right) \sqrt{ }\left[\left(2 t / t_{c 1}-1\right) /\left(2 t / t_{c 2}-1\right)\right] \sim \sqrt{ }\left[t_{c 1} / t_{c 2}\right]=\sqrt{ }\left[h_{c 1} / h_{c 2}\right]=\sqrt{ }\left[w_{1} / w_{2}\right]$.

In conclusion, if we assume that the ion enhances the etch process only at the spots were it collides with the surface then the lag effect will be already pronounced for medium aspect ratios (5-10), even for low pressure RIE equipment with fairly sharp IAD's $\left(\mathrm{FWHM}<5^{\circ}\right)$. Hence, it seems to be effective to sharpen the IAD as much as possible.

\subsection{Image force}

Let's look again at the statement Gottscho gave with respect to RIE lag: "However, further analysis of RIE lag data (Fig.4a) shows that etching rates are not consistent with absolute feature-size scaling but rather aspect ratio scaling [9]. Note that because aspect ratio continually increases during an etch, etching rates are necessarily time-dependent; as the etching proceeds and the aspect ratio grows larger, the etching rate decreases (Fig.4b)". After this he continuous with the image force: "The image force mechanism [8] predicts that RIE lag will increase as feature size decreases. This mechanism cannot be responsible for the observed dependency of etching rates on aspect ratio; however, it is expected to contribute to RIE lag in high aspect ratio, subquarter-micron trenches [8]. "In the next discussion we will use the same data from Chin and co-workers as Gottscho did and compare it with results from the image force theory. But before this, our analytical results will be compared with the numerical output found in the paper by Davis [8].

Davis computed numerically the aspect ratio for which half of the ion flux had collided with the walls due to the image force. We did the same but now the calculation is analytical with first order assumptions. It can be found that the relative etch rate RelR as a function of the trench width $w$, aspect ratio $A R=h / w$, and ion velocity $E_{k i n}$ is [14]:

$$
\operatorname{Re} 1 \mathrm{R}=\frac{\partial \mathrm{h} / \partial \mathrm{t}}{\mathrm{R}_{\max }}=1-\left(\frac{\mathrm{kAR}^{2}}{\mathrm{E}_{\mathrm{kin}} \mathrm{w}}\right)^{1 / 3}
$$

With $k=q / 8 \alpha \pi \varepsilon[V m] \sim 10 \AA V . \alpha \sim 0.7$ accounts for the nonlinear accelaration towards a (solid) medium. The aspect ratio corresponding to the depth at which half of the ion flux into the trench has collided with an etched wall $(\operatorname{RelR}=1 / 2)$ is denoted by $\mathrm{AR}_{1 / 2}$ :

$$
\mathrm{AR}_{1 / 2}=\sqrt{\frac{\mathrm{E}_{\mathrm{kin}} \mathrm{w}}{8 \mathrm{k}}}
$$

The approx. solution of the differential eq. 9 is found to be:

$$
\text { etchtime } \approx \frac{h}{R_{\max }}\left[1+\frac{3}{5 w}\left(\frac{\mathrm{kh}^{2}}{E_{\mathrm{kin}}}\right)^{1 / 3}\right]
$$

and thus:

$$
\text { timelag }=\frac{3}{5 w}\left(\frac{k h^{2}}{E_{k i n}}\right)^{1 / 3}
$$

As can be observed, both graphs match perfectly (Fig.4c, top and bottom). So, it is allowed to use the easier to handle expressions (Eq.9-12) instead of the numerical plots. 

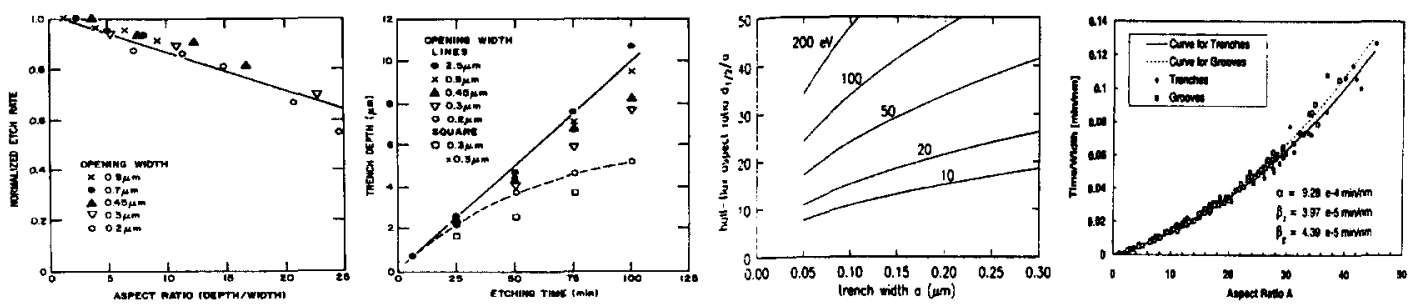

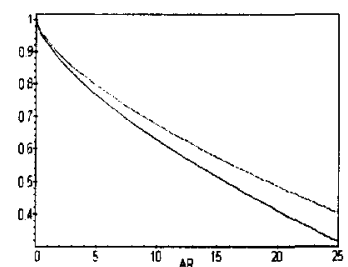

(a)

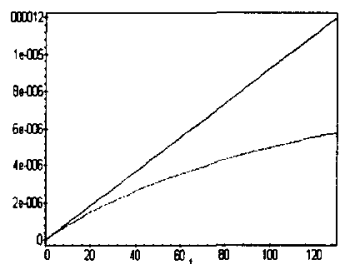

(b)

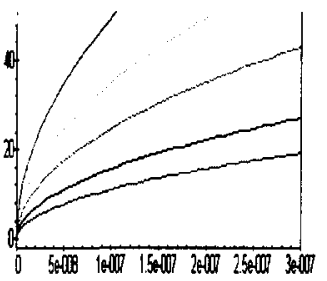

(c)

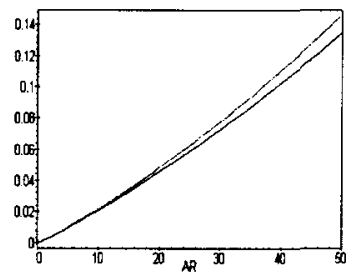

(d)

Figure 4: The image force theory (bottom) compared to experimental data (top) from Chin (a-b), Davis (c), and Müller (d). With the permission of the autors.

Now, let's take the kinetic energy of the incoming ions to be $\mathrm{E}_{\mathrm{kin}}=10 \mathrm{eV}$ and run the Maple program of the image theory, the graphs for all the trenches are matching extremely well with Chin's data as shown in fig. $4 \mathrm{~b}$ for the $0.2[\mu \mathrm{m}]$ and 2.5 $[\mu \mathrm{m}]$ trenches. Quite surprisingly, the same data which was used to exclude the image force mechanism as responsible for the lag effect in micron-sized trenches is used now to make it a possible mechanism. The discrepancy may be caused by the technique used by Chin to obtain fig.4a while using fig.4b [9]. All our attempts to reconstruct fig. $4 \mathrm{a}$ from fig. $4 \mathrm{~b}$ failed and it is unclear for us why: a curved line fit is predicted by the image force and/or ion distribution theory.

Another experimental result supports the image force theory also. Muller and co-workers plotted the etch time $t$ scaled by the feature width $w$ versus the aspect ratio $A R$, as shown in fig. $4 d$-top for differently wide grooves $(w=0.2-$ $0.4 \mu \mathrm{m})$ and trenches $(w=0.2-0.8 \mu \mathrm{m})$. They found that the data points could be fitted by a second order polynomial in AR [15]:

$$
\mathrm{t} / \mathrm{w}=\alpha^{*} \mathrm{AR}+\beta^{*} \mathrm{AR}^{2}
$$

The coefficient $\alpha$ is the inverse of the etch rate for an $A R$ of 0. ... Later on with increasing $A R$, the etch rate is diminished due to RIE lag quantified by the coefficient $\beta$ in eq.13a. This diminution can be different for trenches and grooves due to their different geometrical properties. Therefore we introduced $\beta_{i}$ for trenches and $\beta_{g}$ for grooves. .. trenches and grooves show essentially the same etch rate and RIE lag over the AR regime from 0 to 45. This does not confirm the data of Chin et al. [9], who have observed that grooves are etched approx. $50 \%$ faster than trenches of the same width. However, Chin was discussing square holes whereas Muller used elongated trenches. This is essentially different, not only for the image force theory, but for the distribution theory as well. From the image force theory we may rewrite eq. 11 and get:

$$
\mathrm{t} / \mathrm{w}=\frac{\mathrm{AR}}{\mathrm{R}_{\max }}+\frac{3}{5 \mathrm{R}_{\max }} *\left(\frac{\mathrm{k}}{\mathrm{E}_{\mathrm{kin}} \mathrm{W}}\right)^{1 / 3} * \mathrm{AR}^{5 / 3}
$$

This function is plotted in fig. $4 \mathrm{~d}$-bottom for $10 \mathrm{eV}$ ions,
$R_{\max } \sim 1.1 \mu \mathrm{m} / \mathrm{min}$, and $w=0.2$ and $0.4 \mu \mathrm{m}$ grooves. Both graphs (Fig.4d top-bottom) match reasonable although the curvature is somewhat different. It should be noticed that the distribution theory does give the quadratic behaviour as found in eq. 13 a. After all, rewriting eq. 8 gives:

$$
t / w=\frac{A R_{c}}{2 R_{\max }}+\frac{1}{5 R_{\max } A R_{c}} * A R^{2}
$$

We conclude that the image force can not be excluded as a mechanism responsible for RIE lag in micron-sized HART's.

\subsection{Image force together with the ion distribution}

In this last section we will combine the image force theory together with the ion distribution theory. Let's reconsider the equations for the relative etch rate from the ion distribution theory (Eq.7) and that of the image force theory (Eq.9). The total relative etch rate is the multiplication of both equations:

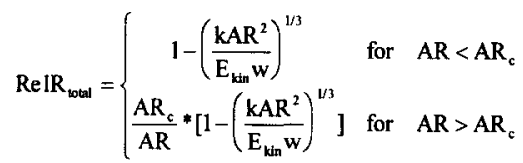

So, for silicon structures etch with the help of the $\mathrm{SF}_{6} / \mathrm{O}_{2}$ ioninhibitor chemistry, the etch rate will follow the feature size dependent image force theory when the aspect ratio is below the critical aspect ratio i.e. $\mathrm{AR}_{\mathrm{c}} \sim 1 / 2 \mathrm{FWHM}$. Beyond that point, the etch rate will follow more and more the aspect ratio dependency of the ion distribution theory.

In fig. 11a the result of the theoretical analysis is shown for trenches etched with $60 \mathrm{eV}$ kinetic ions. The critical aspect ratio is taken as 8 , which corresponds with a $312^{\circ}$ FWHM of the IADF. In fig. $1 \mathrm{lb}$ experimental data is plotted for trenches etched with the help of the ICP-RIE. Although the match is not $100 \%$, the magnitude and the behaviour is quite identical. The match would be better when we would have used a Gauss distribution instead of a triangle function to simulate the ionenergy distribution. As a direct concequence of this approach, an inflection point is observed in the theoretical result at $A R=8$ which is, of course, not found in the experiment. 


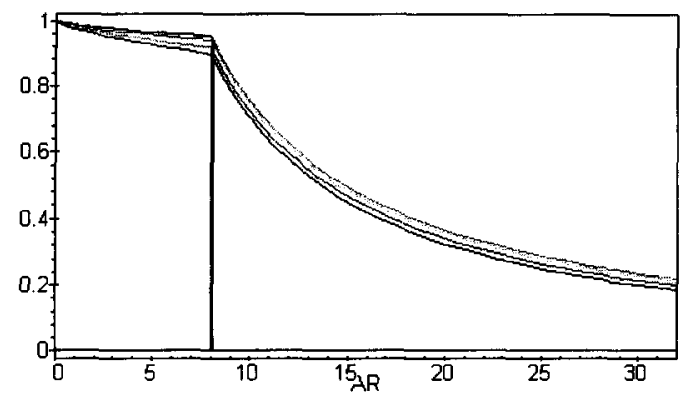

(a)

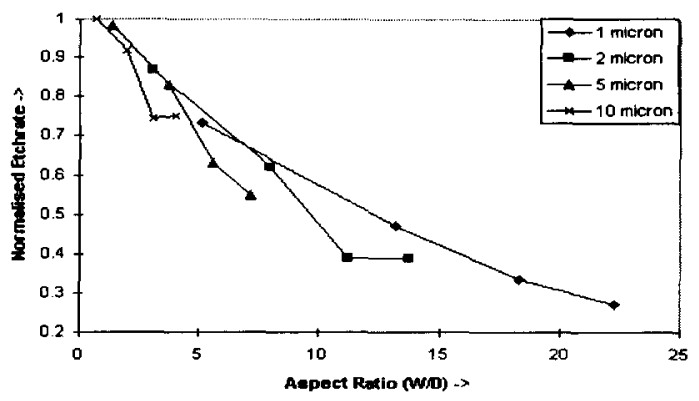

(b)

Figure 11: Relative etch rate of a trench for $w=1,2,5$, and $10 \mu \mathrm{m}, E_{k i n}=60 \mathrm{eV}$, and $A R_{c}=8$. a) Theory and b) experiment.

\section{CONCLUSIONS}

In our quest for the perpetrator of the lag effect into silicon high aspect ratio trenches using an $\mathrm{SF}_{6} / \mathrm{O}_{2} \mathrm{RIE}$ we thought of some experiments which could distinguish between the candidates. There are three main groups to be considered in ion-inhibitor RIE: 1) radicals, 2) inhibitors, and 3) ions.

This paper has given arguments to believe that the image force and the IAD in conjunction with ion shadowing are the important mechanisms to consider for explaining RIE lag. The image force scales with feature size whereas the (triangleshaped) IAD scales inversely with the AR. This gives us the possibility to discriminate between these two mechanisms. However, this is not straightforward. The data from Chin, which was used by Gottscho also, with respect to the AR scaling law does not fit with our results. Nevertheless, there is another way to draw a distinction between the image force and the IAD theory. Trends may be observed with pressure and ion velocity with respect to the lag effect.

The IAD theory predicts that the lag effect increases with pressure. We have written a $\mathrm{c}++$ simulator in windows which had a close match with experimental data and the distribution theory for trenches where the image force is believed to be too small. The simulation shows besides RIE lag the bottling effect (i.e. the trenches are somewhat wider at the top).

The image force theory predicts that the lag effect will decrease when the ion velocity is increased. However, while changing the ion velocity, the ion impact angle is changing too. Unfortunately, it is difficult to separate both effects and to decide which phenomena has the main contribution to the lag effect. So, we have to be careful because the faster the ions, the higher the impact of off-normal ions at the sidewalls. This higher impact energy could make it possible for ions to modify the surface and thus increase the lag effect. In contradiction, it could also be that at higher velocities the ions rebound. The interpretation of the results is even more disturbed because changing the ion velocity by changing the rf power supply will change the sheath thickness also and therefore the FWHM of the IAD. Nevertheless, in comparing the image force theory with the data given by Chin and Muller we found a remarkable good match. So, the image force seems to be a strong candidate to explain the lag effect in micron-sized features. We have to stress that the match is in fact too good; we should have seen the influence of the IAD also. This can be explained because we used our only fitting parameter, the ion velocity, at a value of $10 \mathrm{eV}$ to match experiment with theory. The energy used in the experiments of Chin and Müller was probably higher which would decrease the influence of the image force. Both the image force and the distribution theory together would then result in a satisfying match between theory and experiment. Nevertheless, our point of view with respect to the image force is that you cannot argue with this data that, as Gottscho stressed, "This mechanism cannot be responsible for the observed dependency of etching rates on aspect ratio".

Of course, care should be taken when using simulations. As Gottscho remarked: "In some cases, perhaps only one parameter is varied in fitting the simulation results to experiment. But such comparisons say virtually nothing about the validity of the model and many models are likely to yield comparably good "agreement" with experiment". However, in both our models, the image force theory as well as the ion angular distribution simulation, we didn't make use of any extraordinary fitting parameter. Because of this, the validity of the models is largely enhanced.

In summary: In microengineering, the ion angular distribution is probably the most important mechanism to explain RIE lag. A lower pressure decreases this influence. In nano-engineering, the image force has to be added. Higher energetic ions decreases this effect.

\section{REFERENCES}

[1] A.Hayasaka, Y.Tamaki, M.Kawamura, K.Ogiue, and S.Ohwaki, IEDM Tech.Dig.82, 62 (1982).

[2] H.Sunami, T.Kure, N.Hashimoto, K.Itoh, T.Toyabe, and S.Asai, IEDM Tech.Dig.82, 806 (1982).

[3] M.de Boer, H.Jansen, and M.Elwenspoek, Proc. Eurosensors IX and the 8th international conference on solid-state sensors and actuators, Stockholm, Sweden, 565 (1995).

[4] R.A.Gottscho, C.W.Jurgensen, and D.J.Vitkavage, J.Vac.Sci.Techn. $B 10(5), 2133$ (1992).

[5] H.Jansen, M.de Boer, and M.Elwenspoek, Proc. IEEE micro electro mechanical systems MEMS'96, San diego, California, 250 (1996).

[6] T.M.Mayer and R.A.Barker, J.Vac.Sci.Technol. 21, 757 (1982).

[7] H.Jansen, H.Gardeniers, M.de Boer, M.Elwenspoek, and J.Fluitman, J.of Micromechanics and microengineering, Vol.6, Nol, ISSN: 0960-1317, 14 (1996).

[8] R.J.Davis, Appl.Phys.Lett. 59, 1717 (1991)

[9] D.Chin, S.H.Dhong, and G.J.Long, J.Electroc.Soc. 132, 1705 (1985).

[10] H.Jansen, M.de Boer, J.Burger, R.Legtenberg, and M.Elwenspoek, Microelectronic engineering, Vol.27, 475 (1995).

[11] C.W.Jurgensen, J.Appl.Phys. 64, 590 (1988).

[12] J.Liu, G.L.Huppert, and H.H.Sawin, J.Appl.Phys. 68, 3916 (1990).

[13] J.Janes, J.Vac.Sci.Technol.A 12(1), 97 (1994).

[14] M.Elwenspoek and H.V.Jansen, Etching of Silicon, Cambridge University Press, 40 West 20th Streeth New York, NY, To be published [15] K.P.Muller, K.Roithner, H.J.Timme, Microelec. Engin. 27, 457 (1995). 